\title{
A diffuse-interface model for electrowetting drops in a Hele-Shaw cell
}

\author{
H.-W. LU ${ }^{1}$, K. GLASNER ${ }^{2}$,A. L. BERTOZZI ${ }^{3}$ \\ AND C.-J. K I M ${ }^{1}$ \\ ${ }^{1}$ Department of Mechanical and Aerospace Engineering, UCLA, Los Angeles, CA 90095, USA \\ 2 Department of Mathematics, University of Arizona, Tucson, AZ 85721, USA \\ ${ }^{3}$ Department of Mathematics, UCLA, Los Angeles, CA 90095, USA
}

(Received 22 July 2005 and in revised form 5 July 2007)

Electrowetting has recently been explored as a mechanism for moving small amounts of fluids in confined spaces. We propose a diffuse-interface model for drop motion, due to electrowetting, in a Hele-Shaw geometry. In the limit of small interface thickness, asymptotic analysis shows that the model is equivalent to Hele-Shaw flow with a voltage-modified Young-Laplace boundary condition on the free surface. We show that details of the contact angle significantly affect the time scale of motion in the model. We measure receding and advancing contact angles in the experiments and derive their influence through a reduced-order model. These measurements suggest a range of time scales in the Hele-Shaw model which include those observed in the experiment. The shape dynamics and topology changes in the model agree well with the experiment, down to the length scale of the diffuse-interface thickness.

\section{Introduction}

The dominance of capillarity as an actuation mechanism at the micro-scale has received considerable attention. Darhuber \& Troian (2005) recently reviewed various microfluidic actuators by manipulation of surface tension. Because of the ease of electronic control and low power consumption, electrowetting has become a popular mechanism for microfluidic actuations. The theory of electrowetting and the different applications are well-reviewed by Mugele \& Baret (2005). Lippman (1875) first studied electrocapillary in the context of a mercury-electrolyte interface. The electric double layer is treated as a parallel plate capacitor,

$$
\gamma_{s l}(V)=\gamma_{s l}(0)-\frac{1}{2} c V^{2},
$$

where $\gamma_{s l}$ is the solid-liquid interfacial energy, $c$ is the capacitance per area of the electric double layer, and $V$ is the voltage across the double layer. The potential energy stored in the capacitor is expended in lowering the surface energy. A locally applied voltage then creates the surface energy variation to induce drop motion. Kang (2002) calculated the electro-hydrodynamic forces on a conducting liquid wedge on a perfect dielectric, and recovered equation (1.1).

Dielectric breakdown across the electric double layer limits the applicable voltage. Depositing a thin dielectric film $(\sim 0.1 \mu \mathrm{m})$ using micro-fabrication techniques common in micro electromechanical systems (MEMS) prevents such breakdown without incurring significant voltage penalty (see Moon et al. 2002), making electrowetting-on-dielectric a practical mechanism of micro-scale drop manipulation. 
(a)

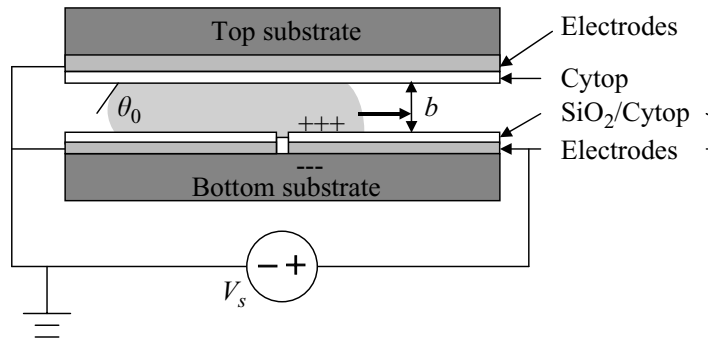

(b)

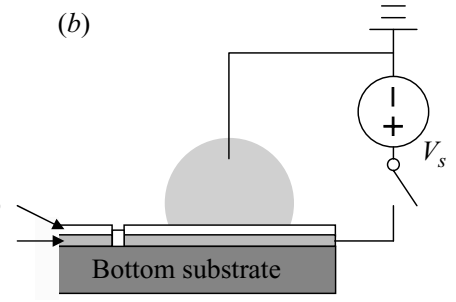

Figure 1. (a) Cross-section of the electrowetting device. (b) Experimental setup for static contact angle measurement.

For such a configuration, $c$ in (1.1) represents the capacitance per area of the dielectric. Lee \& Kim (1998) demonstrated the first working micro-device driven by electrically controlled surface tension with a mercury drop in water. Kim (2000) and Lee et al. (2001, 2002b) subsequently reported handling of water in air. Cho et al. (2001), and Pollack, Fair \& Shenderov (2000) demonstrated cutting, merging, and transport of drops by electrowetting in Hele-Shaw cell geometry. Hayes \& Feenstra (2003) utilized the same principle to produce a video speed display device.

The electrowetting device we consider is shown in figure 1 . The fabrication and the experimental details will be given in the following section. It consists of a fluid drop constrained between two solid substrates separated by a distance, $b$. The aspect ratio of the drop, $\alpha=b / R$, can be controlled by drop volume and substrate separation. Here we consider experiments where $\alpha \leqslant 0.1$. For a constrained drop of radius $R$ much greater than the drop height $b$, the geometry approximates a Hele-Shaw cell (see Hele-Shaw 1898), which enjoys a rich history in fluid mechanics (see Saffman 1986; Homsy 1987; Bensimon et al. 1986; Howison 1992; Tanveer 2000).

For partially wetting fluids inside a Hele-Shaw cell, surface effects at the contact line can influence the interfacial boundary conditions. Paterson, Fermigier \& Limat (1995) and Paterson \& Fermigier (1997) experimentally studied the influence of strong surface defects on the interface of silicon oil and water displacing air in a Hele-Shaw cell. Carrillo, Soriano \& Ortin $(1999,2000)$ found that the fingering instability and the displacement speed of an expanding fluid annulus in a rotating Hele-Shaw cell is highly sensitive to the wetting condition of the outer interface. Weinstein, Dussan V. \& Ungar (1990) theoretically investigated the influence of dynamic contact angles on the steady-state solutions of viscous fingering in a Hele-Shaw cell. The dynamic contact angle is incorporated into the pressure boundary condition but has only geometric dependence on the steady finger shape. The analysis is specific to a travelling wave solution and does not directly carry over to the general flow problem in Hele-Shaw cell.

Owing to the intense interest in the Hele-Shaw problems, the last ten years has also seen the development of numerical methods for solving the Hele-Shaw problem. The boundary integral method developed by Hou, Lowengrub \& Shelley (1994) has been quite successful in simulating the long time evolution of free-boundary fluid problems in a Hele-Shaw cell. However, simulating drops that undergo topological changes remains a complicated, if not ad hoc, process for methods based on sharp interfaces. Diffuse interface models provide alternative descriptions by defining a phase-field variable that assumes a distinct constant value in each bulk phase. The 
material interface is considered as a region of finite width in which the phase-field variable varies rapidly but smoothly from one phase value to another. Such diffuseinterface methods naturally handle topology changes. As we demonstrate in this paper, an energy construction provides a convenient framework in which to incorporate a spatially varying surface energy due to electrowetting. Matched asymptotic expansions may be used to demonstrate the equivalence between the diffuse-interface dynamics and the sharp-interface dynamics in the Hele-Shaw cell.

In this paper we develop a diffuse-interface framework for the study of HeleShaw cell drops that undergo topological changes by electrowetting. Using level-set methods, Walker \& Shapiro (2006) considered the unsteady problem of a drop deformed by a rapidly changing potential distribution. We consider a viscous flow induced by a steady potential. The related work of Lee, Lowengrub \& Goodman (2002a) considered a diffuse interface in the absence of electrowetting in a Hele-Shaw cell under the influence of gravity. They used a diffuse-interface model for the chemical composition, coupled to a classical fluid dynamic equation. Our model describes both the fluid dynamics and the interfacial dynamics through a nonlinear Cahn-Hilliard equation of one phase-field variable. Our approach expands on the work done by Glasner (2003) and is closely related to that of Kohn \& Otto (1997) and Otto (1998).

Section 2 describes the experimental setup of drop manipulation using electrowetting-on-dielectric. Section 3 describes the sharp-interface fluidic model for the electrowetting drop in Hele-Shaw cell. We also briefly discuss the role of the contact line in the context of the sharp-interface model. In $\S 4$, we describe the diffuse-interface model of the problem. Equivalence with the sharp-interface model will be accomplished in $\S 5$ through matched asymptotic expansions. Comparisons will be made in $\S 6$ between the experimental data and the numerical results. To relate the theoretical prediction to the experimental observation, we construct a reducedorder model in $\S 7$ with constant contact angles similar to the contact angle models discussed in Saffman (1986), followed by conclusions.

\section{Experimental setup}

\subsection{Procedure}

The fabrication of electrowetting devices is well documented in Cho, Moon \& Kim (2003). Unlike the previous work, we enlarge the device geometry by a factor of 10 and use a more viscous fluid such as glycerine to maintain the same Reynolds number as in the smaller devices. Such a modification allows us to more carefully maintain the substrate separation and to directly measure the contact angles. The devices are fabricated in the UCLA Nanoelectronics Research Facility. The bottom substrate is patterned with square metal electrodes of size $1 \mathrm{~cm}$. Two dielectric layers are placed

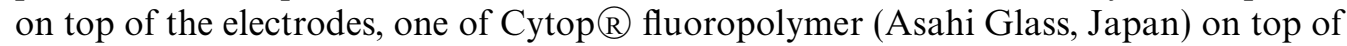
a layer of silicon dioxide $\left(\mathrm{SiO}_{2}\right)$. The top substrate is a cleaved glass with a conductive

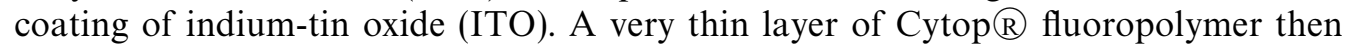
covers the ITO to increase the surface energy. The thicknesses of the materials used in the device are summarized in table 1. A drop of fluid with $\alpha \sim 0.1$ is dispensed on the bottom substrate. Two solid spacers maintain the substrate separation at $\sim 530 \mu \mathrm{m}$ (see figure $1 a$ ).

If the top substrate is the same as the bottom substrate, the potential drop across its dielectric layers will involve the electrical properties of the fluid. To simplify the

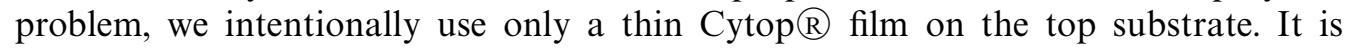
well-know that a thin fluoropolymer film is a poor insulator (see Seyrat \& Hayes 


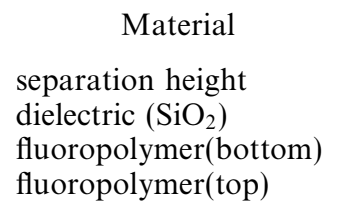

$$
\begin{gathered}
\text { Thickness } \\
529 \pm 2 \mu \mathrm{m} \\
498.4 \pm 15.5 \mathrm{~nm} \\
245.8 \pm 15.5 \mathrm{~nm} \\
50 \mathrm{~nm}
\end{gathered}
$$

Dielectric constant

$$
\begin{gathered}
\mathrm{n} / \mathrm{a} \\
3.8 \\
2.0 \\
2.0
\end{gathered}
$$

TABLE 1. Material thicknesses and dielectric constants of an electrowetting device.

2001) so it only affects the charging time across the thick dielectric layers on the bottom substrate. We conduct a set of static experiments using deionized water with surface tension of $72 \mathrm{dyn}^{-1}$ to confirm this. First, the capacitance per area of the $\mathrm{SiO}_{2} /$ Cytop layers on the bottom substrate is characterized by measuring the static contact angle of a sessile drop of deionized water under the influence of a varying voltage (see figure $1 b$ ). Then we cover the drop with the top substrate and repeat the experiment to characterize the electrowetting effect across the thin Cytop film on the top substrate.

The glycerine-water mixture with viscosity of $0.020 \mathrm{~Pa} \mathrm{~s}$, density of $1.28 \mathrm{~cm}^{-3}$, and surface tension of $67 \mathrm{dyn} \mathrm{cm}^{-1}$ is used for the dynamic experiments. The half-height of the cell is an order of magnitude smaller than the capillary length, $\sqrt{\gamma / \rho g} \approx 2600 \mu \mathrm{m}$, preserving the dominance of surface tension over gravity. To move a drop, part of the drop interface must extend over an adjacent electrode. The top substrate and the electrode below the drop are grounded. Applying a potential on an adjacent electrode moves the drop to that electrode. The voltage level is varied between $30 \mathrm{~V} \mathrm{DC}$ and 80 V DC. At each voltage level, we move a drop back and forth between two electrodes ten times by alternately applying the voltage and ground to each electrode. A drop can be split by applying potentials on both sides of the drop. A camera is used to record the motion, from above, at 30 frames per second. Side views of the menisci at the nose and the tail of a translating drop are recorded at $512 \times 256$ resolution by a high-speed camera (Vision Research Inc., Wayne, NJ) at 2100 frames per second. The slope of the top substrate is known a priori by focusing on its cleaved edge. The bottom substrate is assumed to have the same slope. Both surfaces create reflections of the menisci allowing us to locate the contact lines. The images are processed by

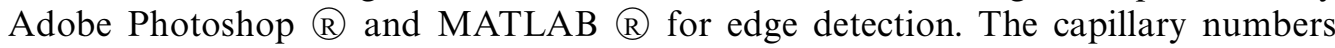
of the advancing and receding contact lines are obtained from the side view and the top view of the drop. Time-indexed images of the translating and splitting drop are compared to the simulation contours in $\S 6$.

\subsection{Statics}

Using Young's equation, we relate the change of contact angle to the electrowetting voltage,

$$
\cos \theta_{V}-\cos \theta_{0}=\frac{c V^{2}}{2 \gamma_{l v}},
$$

where $\theta_{V}$ is the static contact angle when we apply a potential, $V$, on the electrodes. $\theta_{0}$ is the contact angle at zero potential, and $\gamma_{l v}$ is the liquid-vapour surface tension. Figure 2 shows that $\cos \theta_{V}$ linearly increases as a function of $V^{2}$, below a critical voltage. Above the critical voltage, the contact angle saturates and even decreases. This behaviour is in agreement with previous studies (see Verheijen \& Prins 1999; Peykov, Quinn \& Ralston 2000; Seyrat \& Hayes 2001; Vallet, Vallade \& Berge 1999; Shapiro 


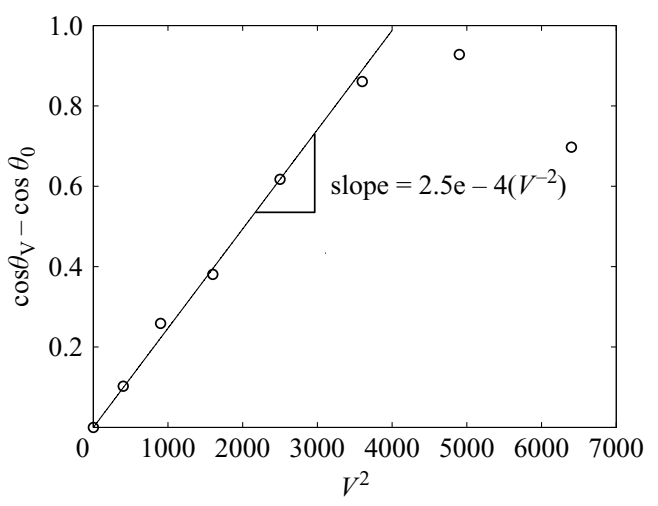

Figure 2. $\cos \theta_{V}-\cos \theta_{0}$ vs. $V^{2}$.

(a)

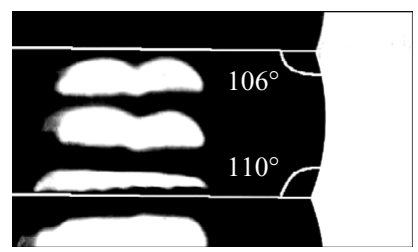

(a)

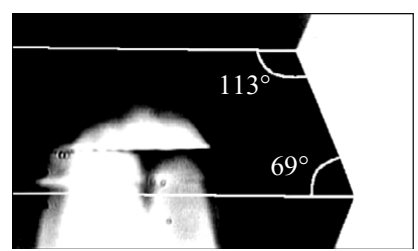

FIGURE 3. Static contact angles $\left( \pm 2^{\circ}\right)$ of a deionized water drop in an electrowetting device (a) before applying voltage, and (b) after application of $50.18 \mathrm{~V}$.

et al. 2003). The model developed in this work will focus on the voltage level below the saturation. The capacitance per area of the $\mathrm{SiO}_{2} /$ Cytop layers $c=3.6 \times 10^{-5} \mathrm{~F}$ is obtained by multiplying the slope of the linear region with $2 \gamma_{l v}$. Estimating this quantity by considering the dielectric layers as two capacitors connected in series (see Moon et al. 2002) gives a value close to that measured experimentally.

Figure 3 shows the static contact angles of a deionized water drop in an electrowetting device with a top substrate both with and without an applied voltage. The increase of the top contact angle is of the same order of magnitude as the uncertainty in the measurement. Therefore, we ignore this small increase in our model and instead focus on the dramatic decrease of the contact angle, by about $40^{\circ}$, on the bottom substrate.

\subsection{Dynamics}

The average velocity of the glycerine--water drop is obtained by dividing the length travelled by the time. Figure 4, inset $(a)$ shows that the drop remains static until a threshold voltage is reached. The threshold voltage to move a glycerine-water drop is approximately $38 \mathrm{~V}$ DC. The interface motion remains smooth for voltages up to $65 \mathrm{~V} \mathrm{DC}$ as shown in figure 4 , inset $(b)$. The average velocity of the drop, $\bar{u}$, increases linearly with the square of the applied voltage. The typical drop velocity $(\bar{u} \approx 0.1-1 \mathrm{~cm})$ indicates small scaled Reynolds number, $R e^{*}=\alpha^{2} \times \rho \bar{u} R / \mu \sim O\left(10^{-2}\right)$. At a higher voltage, we observed pinning on a section of the advancing contact line, causing the drop to form asymmetric shapes such as the one shown in figure 4, inset (c). When moving a drop back and forth between two electrodes using such a high voltage, each drop movement becomes slower than the previous one. Eventually the drop stops responding to the applied voltage, suggesting an accumulation of charges trapped in the dielectric layers. 


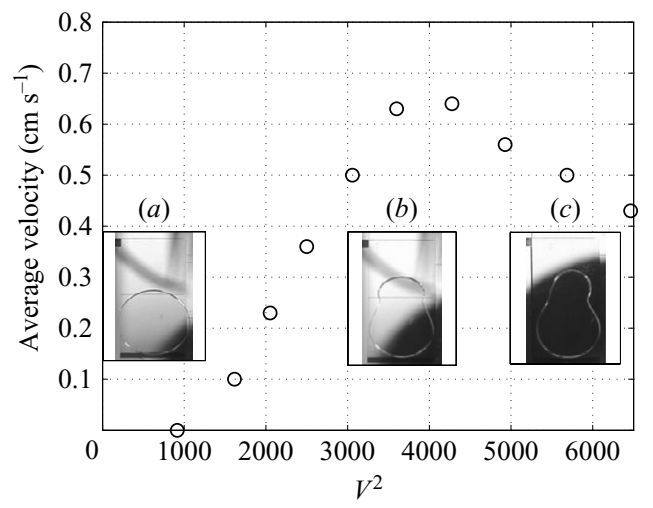

FIGURE 4. Average velocity of the drop actuated by electrowetting. Insets show $(a)$ the drop remains motionless at low voltage level $(30.2 \mathrm{~V} \mathrm{DC}) ;(b)$ the drop advances smoothly at 50.4 V DC; $(c)$ asymmetric shape develops due to the pinning of the advancing contact line at 80.0 V DC.

Time $=0 \mathrm{~ms}$

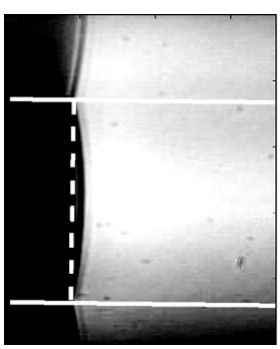

Time $=7.62 \mathrm{~ms}$

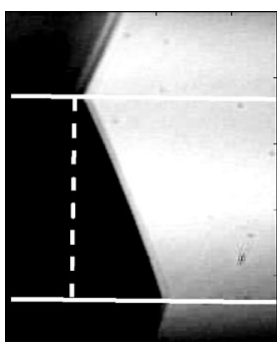

$1.90 \mathrm{~ms}$

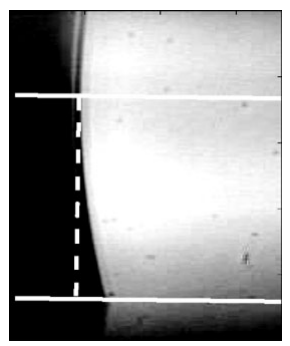

$15.7 \mathrm{~ms}$

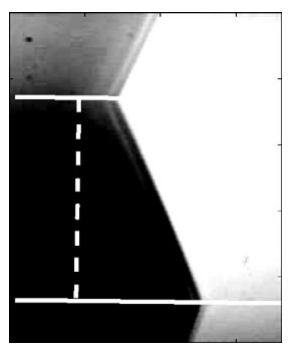

$3.81 \mathrm{~ms}$

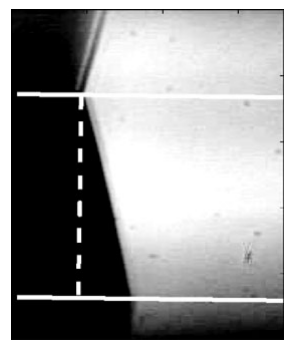

$32.4 \mathrm{~ms}$

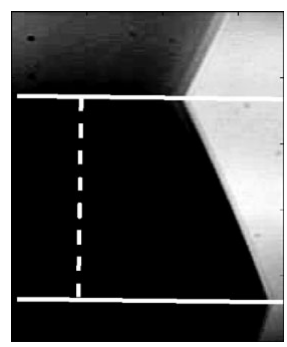

FIGURE 5. Side view of the advancing meniscus of a glycerine-water drop (dark area) at 50.28 V DC. The solid white lines depict the orientations of the top and bottom substrates. Above and below the white lines are the reflections of the meniscus. The dashed line shows the initial position of the meniscus.

To fully understand the problem we must also measure the effect of electrowetting on the dynamic contact angles of the drop. A side view of the advancing meniscus is shown in figure 5 . The locations of the solid surfaces are depicted by two solid white lines. A dashed line intersecting the substrates indicates the initial positions of the contact lines. The onset of electrowetting induces a sudden decrease of the contact angle on the bottom substrate from its static value $\approx 100^{\circ}$, as shown in figure 6 . The capillary numbers of the contact lines, shown in figure 7, quickly settle down to the 

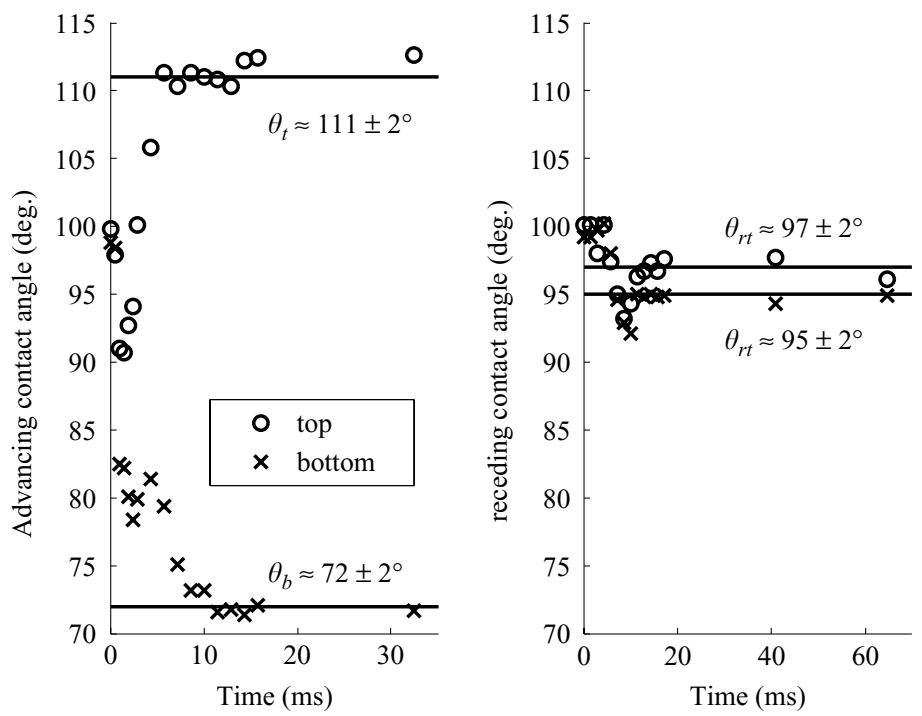

FIGURE 6. Evolution of the advancing and receding contact angles of the meniscus in figure 5.

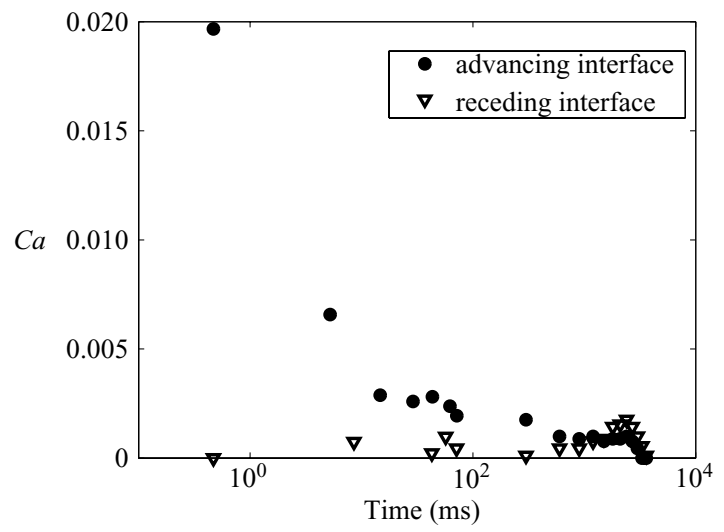

FIGURE 7. Capillary number of the advancing and receding meniscus.

range of $\mathrm{Ca} \sim 10^{-3}$. The contact line on the top substrate remains static until an angle of advance $\approx 110^{\circ}$ is exceeded. The hysteretic effect causes the initially concave meniscus to become convex. The contact angles converge toward steady-state values after a short transient time. The steady behaviour of the contact angle is consistent with the recent numerical finding of Yeo \& Chang (2006) for electrowetting films. The direct observation by the camera only reveals the contact angles at two points of the curvilinear interface. Along the interface, the capillary number varies with the normal velocity of the interface. Therefore, we expect the dynamic contact angle to vary along the interface. More advanced experimental techniques are required to characterize the evolution of the dynamic contact angle on the entire interface.

\section{Sharp-interface description}

Here we review the classical model of Hele-Shaw fluid dynamics and extend the model to include electrowetting. For simplicity, we will first neglect the presence of the 
contact line. The insight into the fluid dynamics will allow us to construct a simple leading-order contact line model in $\S 7$.

Consider a viscous drop occupying a space $[\Omega \times b]$, where $b$ is the height of the Hele-Shaw cell. The small value of aspect ratio allows us to employ the lubrication theory to reduce the momentum equation to Darcy's law coupled with a continuity equation,

$$
\begin{aligned}
\boldsymbol{U} & =-\frac{b^{2}}{12 \mu} \nabla P, \\
\nabla \cdot \boldsymbol{U} & =0,
\end{aligned}
$$

where $\boldsymbol{U}$ is the depth-averaged velocity, $P$ is the pressure in the drop or the bubble, and $\mu$ is the viscosity. Equations (3.1) and (3.2) imply that the pressure is harmonic, $\Delta P=0$.

The interfacial velocity is the fluid velocity normal to the interface, i.e. $\left.\boldsymbol{U}_{n} \sim \nabla P\right|_{\partial \Omega} \cdot \hat{\boldsymbol{n}}$. The boundary condition for normal stress depends on the interactions between the different dominant forces in the meniscus region. Assuming pressure in the surrounding fluid is zero:

$$
\left.P\right|_{\partial \Omega}=\gamma_{l v}\left(A \kappa_{0}+B \kappa_{1}\right)
$$

$\kappa_{0}$, defined as $1 / r$, is the local horizontal curvature and $\kappa_{1}$ is defined as $2 / b$. Different dynamics and wetting conditions at the meniscus determine the actual curvatures of the drop through $A$ and $B$. In their initial study, Saffman \& Taylor (1959) made the assumptions that $A=1$ and $B=-\cos \theta_{A}$ where $\theta_{A}$ is the apparent contact angle measured from inside the drop or bubble.

For the incomplete displacement of viscous fluid, Bretherton (1961) showed that $B=1+\beta C a^{2 / 3}$ where $\beta$ is equal to 3.8 and -1.13 for advancing and receding menisci respectively. Park \& Homsy (1984) and Reinelt (1987) further showed that $A=\pi / 4+O\left(\mathrm{Ca}^{2 / 3}\right)$. For the special case of a complete displacement by a steady-state finger as considered by Weinstein et al. (1990), the boundary condition can also be formulated in the form of equation (3.3). In this case, $B=-\cos \left(\Theta_{R}-\sigma \cos \Phi\right)$ where $\Theta_{R}$ is the angle of recede for the displaced fluid, $\sigma=\partial \Theta / \partial U$ is a linear model for the dynamic contact angle, and $\Phi$ is the angle between the outward normal of the steady-state finger and the velocity $U$. For a partially wetting drop, we assume that $A=1$, which is supported a posteriori in Appendix B.

When a voltage is applied across an electrode, $V(x)=V \chi(x)$, where $\chi(x)$ is a characteristic function of the electrode, $\Omega_{w}$, it locally decreases the solid-liquid surface energy inside the region $\Omega_{w} \bigcap \Omega$ :

$$
\gamma_{w}(V)=\gamma_{l v}\left(-\cos \theta_{0}-\frac{c V^{2}}{2 \gamma_{l v}}\right),
$$

where $\gamma_{w}(V)$ is the difference between the liquid-solid and the solid-vapour surface energy. In deriving (3.4), we assume that the electrowetting does not affect the solidvapour surface energy. From the experimental observations, only the dielectric layer on the bottom substrate produces a significant electrowetting effect. Therefore, the solid-liquid surface energy of a drop in the device is

$$
\gamma_{d e v}=\gamma_{l v}\left(-2 \cos \theta_{0}-\frac{c V^{2}}{2 \gamma_{l v}}\right) .
$$




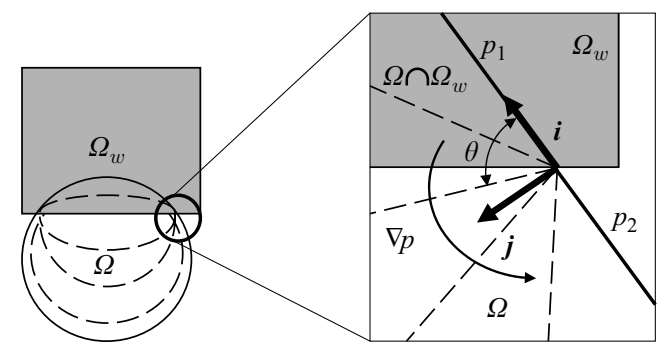

FIGURE 8. Illustration of electrowetting acting on a drop and the details near the boundary of the electrode. Dashed lines depict the pressure contours.

The dominance of surface tension allows us to assume a circular profile for the liquid-vapour interface. Substituting (2.1) into (3.5) gives

$$
\gamma_{d e v}=\gamma_{l v}\left(-\cos \theta_{0}-\cos \theta_{V}\right)=\gamma_{l v} b \kappa_{w},
$$

where $\kappa_{w}=\left(-\cos \theta_{0}-\cos \theta_{V}\right) / b$ is the curvature of the cross-substrate interface in the presence of electrowetting. The constant $\cos \theta_{0}$ does not affect the dynamics, so we will consider it to be zero. Equating $\kappa_{w}=B \kappa_{1}$ shows $B=-c V^{2} / 4 \gamma_{l v}$.

For a drop of volume $v$ placed inside a Hele-Shaw cell with plate spacing of $b$, the radius is $R=(v / \pi b)^{1 / 2}$. We non-dimensionalize the Hele-Shaw equations by the following scales:

$$
r \sim R \tilde{r}, \quad t \sim \frac{12 \mu R}{\gamma_{l v} \alpha^{2}} \tilde{t}, \quad P \sim \frac{\gamma_{l v}}{R} \tilde{P}, \quad(x, y, z) \sim(R, R, b) .
$$

Removing the tilde gives the following equations in dimensionless variables:

$$
\begin{aligned}
\Delta P & =0, \\
\boldsymbol{U} & =-\nabla P, \\
\left.P\right|_{\partial \Omega} & =A \kappa_{0}+B \kappa_{1}, \\
\boldsymbol{U}_{n} & =\left.\nabla P\right|_{\partial \Omega} \cdot \hat{\boldsymbol{n}} .
\end{aligned}
$$

Without the applied voltage, $V=B=0$, the drop relaxes by surface tension. The constant $A$ can then be incorporated into the relaxation time scale. Therefore, the classical relaxation of Hele-Shaw droplets has no dimensionless parameters, meaning that all drops starting from similar initial conditions can be collapsed to the same problem in dimensionless form.

Electrowetting introduces a discontinuous change of the capillary pressure, $\left(P_{2}-\right.$ $\left.P_{1}\right)=-B \kappa_{1}$, as shown in figure 8 . Solving the Laplacian pressure and the associated velocity near the discontinuity gives

$$
\begin{aligned}
P & =-\frac{B \kappa_{1}}{\pi} \arctan \left(\frac{y}{x}\right)+P_{1}, \\
U & =-\nabla P=\frac{B \kappa_{1}}{\pi} \frac{(-y \hat{\boldsymbol{i}}+x \hat{\boldsymbol{j}})}{x^{2}+y^{2}} .
\end{aligned}
$$

where $\hat{i}$ and $\hat{j}$ are the unit axes of a locally orthogonally coordinate system. Near $\partial \Omega_{w}$, electrowetting pumps the fluid into the wetting region. Therefore $B \kappa_{1}$ dictates the time scale of the motion. Away from $\partial \Omega_{w}$, where $B \kappa_{1}$ is relatively constant, the drop relaxes to minimize the liquid-vapour interface and $A \kappa_{0}$ influences this relaxation time scale. This interaction introduces one dimensionless parameter to the classical 
Hele-Shaw flow: we define the electrowetting number

$$
\omega=-B \kappa_{1}=\frac{c V^{2}}{2 \alpha \gamma_{l v}}
$$

as the relative measure between the electrowetting potential and the total energy of the liquid-vapour interface. This parameter affects both the time scale of motion and the time scale associated with the drop morphology.

In applying the electrowetting model (3.14) for $B \kappa_{1}$, we assume the contact angles are determined from a quasi-static balance between the surface energies and the electrical potential. The presence of moving contact lines introduces deviations from the equilibrium values and reduces capillary pressure, $B \kappa_{1}$. In $\S 7$, we will introduce the complication of contact line dynamics through the local dependence of $A$ and $B$ on the contact angles.

\section{Diffuse-interface model}

Diffuse-interface (phase-field) models have the advantage of automatically capturing topological changes such as drop splitting and merger. Here we extend Glasner's (2003) diffuse-interface model to include electrowetting. The model begins with a description of the surface energies in terms of a 'phase' function $\rho$ that describes the depth average of fluid density in a cell. Therefore $\rho=1$ corresponds to fluid and $\rho=0$ to vapour. Across the material interface, $\rho$ varies smoothly over a length scale $\epsilon$.

The total energy is given by the functional

$$
E(\rho)=\int_{\Omega} \frac{A}{\gamma}\left(\frac{\epsilon}{2}|\nabla \rho|^{2}+\frac{g(\rho)}{\epsilon}\right)-\rho \omega \mathrm{d} x .
$$

The first two terms of the energy functional approximate the total liquid-vapour surface energy $\int_{\partial \Omega} \gamma \mathrm{d} S$ where $\partial \Omega$ is the curve describing the limiting sharp interface. An interface between liquid and vapour is established through a competition between the interfacial energy associated with $|\nabla \rho|^{2}$ and the bulk free energy $g(\rho)$ that has two equal minima at $\rho_{l}$ and $\rho_{v}$. To avoid the degeneracy in the resulting dynamic model (see equations (4.7)-(4.8) and to maintain consistency with the desired sharpinterface limit, we choose $\rho_{l}=1$ and $\rho_{v}=\epsilon$. The final term $\rho \omega$ accounts for the wall energy (the difference between the solid-liquid and solid-vapour surface energies) on the solid plates. The first two terms act as line energies around the boundary of the drop while the third term contributes the area energy of the solid-liquid interfaces.

In equation (4.1), $\gamma$ is a normalization parameter which we discuss below. A one-dimensional equilibrium density profile can be obtained by solving the EulerLagrange equation of the leading-order energy functional in terms of a scaled spatial coordinate, $z=x / \epsilon$,

$$
\left(\rho_{0}\right)_{z z}-g^{\prime}\left(\rho_{0}\right)=0
$$

which has some solution $\phi(z)$ independent of $\epsilon$ that approaches the two phases $\rho_{l}, \rho_{v}$ as $z \rightarrow \pm \infty$. Integrating equation (4.2) once gives

$$
\frac{\epsilon}{2} \phi_{x}^{2}=\frac{g(\phi)}{\epsilon} .
$$


Equation (4.3) implies equality between the first and second terms of the energy functional so the total liquid-vapour interfacial energy can be written as

$$
\gamma=\int_{-\infty}^{\infty}(\phi)_{z}^{2} \mathrm{~d} z=2 \int_{-\infty}^{\infty} g(\phi) \mathrm{d} z
$$

Equation (4.4) indicates that the choice of $g(\phi)$ used to model the bulk free energy influences the amount of interfacial energy in the model. Hence this constant appears as a normalization parameter in the first two terms of the energy functional (4.1).

Since there is no inertia in the physical system, the dynamics take the form of a generalized gradient flow of the total energy, which can be equivalently characterized as a balance between energy dissipation and the rate of free energy change,

$$
D \approx \int_{R^{2}} \rho|\boldsymbol{U}|^{2} \mathrm{~d} x \mathrm{~d} y .
$$

Since $\rho$ is conserved, $\rho_{t}=-\nabla \cdot(\rho \boldsymbol{U})$. Using this fact and equating the rate of energy dissipation to the rate of energy change gives

$$
\int_{R^{2}} \rho|\boldsymbol{U}|^{2} \mathrm{~d} x \mathrm{~d} y=-\int_{R^{2}} \rho_{t} \delta E \mathrm{~d} x \mathrm{~d} y=-\int_{R^{2}} \rho \nabla(\delta E) \cdot \boldsymbol{U} \mathrm{d} x \mathrm{~d} y .
$$

To make this true for an arbitrary velocity field $\boldsymbol{U}$, it follows that $\boldsymbol{U}=-\nabla(\delta E)$. Substituting the velocity back into the continuity gives the evolution of the fluid density,

$$
\begin{aligned}
& \epsilon \rho_{t}=\nabla \cdot(\rho \nabla(\delta E)), \\
& \delta E=\frac{A}{\gamma}\left(-\epsilon^{2} \Delta \rho+g^{\prime}(\rho)\right)-\epsilon \omega,
\end{aligned}
$$

subject to boundary conditions that requires no surface energy and no flux at the domain boundary,

$$
\begin{array}{r}
\nabla \rho \cdot \hat{\boldsymbol{n}}=0, \\
\rho \nabla(\delta E) \cdot \hat{\boldsymbol{n}}=0 .
\end{array}
$$

Equations (4.7)-(4.8) with $\omega=0$ constitute a fourth-order Cahn-Hilliard equation with a degenerate mobility term. By letting $\omega$ having spatial dependence, we introduce electrowetting into the diffuse-interface model.

\section{Asymptotic analysis}

Matched asymptotic expansions show that the sharp-interface limit of the constantmobility Cahn-Hilliard equation approximates the two-side Mullins-Sekerka problem (see Caginalp \& Fife 1988; Pego 1989). The recent work of Glasner (2003) showed that the degenerate Cahn-Hilliard equation approaches the one-sided Hele-Shaw problem in the sharp-interface limit. Using a similar method, we show that the sharp-interface limit of the modified Cahn-Hilliard equation (4.7)-(4.8) recovers the Hele-Shaw problem with electrowetting (3.8)-(3.11). The diffuse-interface approximation allows us to simulate topology changes without artificial surgery of the contour. This is especially useful as electrowetting devices are designed for the purpose of splitting, merging and mixing of drops.

Using a local orthogonal coordinate system $(z, s)$, where $s$ denotes the distance along $\partial \Omega$ and $z$ denotes signed distance to $\partial \Omega, r$, scaled by $1 / \epsilon$. The dynamic 
equation expressed in the new coordinates is

$$
\begin{aligned}
\epsilon^{2} \bar{\rho}_{z} r_{t}+\epsilon^{3}\left(\bar{\rho}_{s} s_{t}+\bar{\rho}_{t}\right)= & \left(\bar{\rho}(\delta \bar{E})_{z}\right)_{z}+\epsilon \bar{\rho}(\delta \bar{E})_{z} \Delta r \\
& +\epsilon^{2}\left[\bar{\rho}(\delta \bar{E})_{s} \Delta s+\left(\bar{\rho}(\delta \bar{E})_{s}\right)_{s}|\nabla s|^{2}\right], \\
\delta \bar{E}= & \frac{A}{\gamma}\left(-\bar{\rho}_{z z}-\epsilon \bar{\rho}_{z} \Delta r-\epsilon^{2}\left(\bar{\rho}_{s s}|\nabla s|^{2}+\bar{\rho}_{s} \Delta s\right)+g^{\prime}(\bar{\rho})\right)-\epsilon \omega
\end{aligned}
$$

where the ${ }^{-}$denotes the variables in the inner region. The matching conditions are

$$
\begin{aligned}
& \bar{\rho}^{(0)}(z) \sim \rho^{(0)}( \pm 0), z \rightarrow \pm \infty, \epsilon z \rightarrow \pm 0, \\
& \bar{\rho}^{(1)}(z) \sim \rho^{(1)}( \pm 0)+\rho_{r}^{(0)}( \pm 0) z, z \rightarrow \pm \infty, \epsilon z \rightarrow \pm 0, \\
& \bar{\rho}^{(2)}(z) \sim \rho^{(2)}( \pm 0)+\rho_{r}^{(1)}( \pm 0) z+\rho_{r r}^{(0)}( \pm 0) z^{2}, z \rightarrow \pm \infty, \epsilon z \rightarrow \pm 0 .
\end{aligned}
$$

Similar conditions can be derived for $\delta \bar{E}$ and $\delta E$.

The $O(1)$ inner expansion gives

$$
\begin{aligned}
\left(\bar{\rho}^{(0)}\left(\delta \bar{E}^{(0)}\right)_{z}\right)_{z} & =0, \\
\frac{A}{\gamma}\left(g^{\prime}\left(\bar{\rho}^{(0)}\right)-\bar{\rho}_{z z}^{(0)}\right) & =\delta \bar{E}^{(0)} .
\end{aligned}
$$

Equation (5.4) implies $(\delta E)^{(0)}=C(s, t)$. Equation (5.5) is the equation for the onedimensional steady state. The common tangent construction implies

$$
\delta \bar{E}^{(0)}=\frac{\gamma\left(g\left(\rho_{l}\right)-g\left(\rho_{v}\right)\right)}{A\left(\rho_{l}-\rho_{v}\right)} .
$$

The double-well structure of $g(\bar{\rho})$ implies $(\delta \bar{E})^{(0)}=0$. At the leading order, the competition between interfacial energy and the bulk free energy establishes a stable diffuse interface.

The $O(1)$ outer expansion of (4.7), (4.8) gives

$$
\nabla \cdot\left(\rho^{(0)} \nabla g^{\prime}\left(\rho^{(0)}\right)\right)=0 .
$$

The unique solution in the dense phase that satisfies no-flux and matching conditions is constant, $\rho^{(0)}=\rho_{l}$. Thus on an $O(1)$ scale no motion occurs.

The $O(\epsilon)$ inner expansion results in

$$
\begin{aligned}
\left(\bar{\rho}^{(0)}\left(\delta \bar{E}^{(1)}\right)_{z}\right)_{z} & =0, \\
\frac{A}{\gamma}\left(g^{\prime \prime}\left(\bar{\rho}^{(0)}\right)-\frac{\partial^{2}}{\partial z^{2}}\right) \bar{\rho}^{(1)} & =\delta \bar{E}^{(1)}-\frac{A}{\gamma} \kappa^{(0)} \bar{\rho}_{z}^{(0)}+\omega,
\end{aligned}
$$

where the leading-order curvature in the horizontal plane, $\kappa^{(0)}$, is identified with $-\Delta r$. Applying matching boundary condition for $\delta \bar{E}^{(1)}$ to equation (5.8) shows that $\delta \bar{E}^{(1)}$ is independent of $z ; \bar{\rho}^{(1)}=\bar{\rho}_{z}^{(0)}$ is the homogenous solution of (5.9). In the region of constant $\omega$, the solvability condition gives

$$
\rho_{l}\left(\delta \bar{E}^{(1)}\right)=\frac{A \kappa^{(0)}}{\gamma} \int_{-\infty}^{\infty}\left(\bar{\rho}_{z}^{(0)}\right)^{2} \mathrm{~d} z-\omega \rho_{l} .
$$

The integral is equal to $\gamma$, using (4.4). Assuming $\rho_{l}=1$ and using (3.14) gives

$$
\delta \bar{E}^{(1)}=\left(A \kappa^{(0)}+B \kappa_{1}\right) .
$$

The surface energy term includes both curvatures of the interface. This is analogous to the Laplace-Young condition of a liquid-vapour interface. 
In regions where sharp variation of $\omega$ intersects the diffuse interface, the solvability condition becomes

$$
\rho_{l}\left(\delta \bar{E}^{(1)}\right)=A \kappa^{(0)}-\int_{-\infty}^{\infty} \omega \bar{\rho}_{z}^{(0)} \mathrm{d} z .
$$

The sharp surface energy variation is smoothly weighted by $\bar{\rho}_{z}^{(0)}$, which is $O(1)$ for a phase function $\bar{\rho}$ that varies smoothly between 0 and 1 in the scaled coordinate.

To order $\epsilon$, the outer equation in the dense phase must solve

$$
\Delta\left(\delta E^{(1)}\right)=0,
$$

with a no-flux boundary condition in the far field, and a matching condition at the interface described by (5.2).

The $O\left(\epsilon^{2}\right)$ inner expansion reveals the front movement

$$
U_{n}^{(0)} \bar{\rho}_{z}^{(0)}=\left(\bar{\rho}^{(0)}\left(\delta \bar{E}^{(2)}\right)_{z}\right)_{z},
$$

where $r_{t}$ is identified as the leading-order normal velocity of the interface $U_{n}^{(0)}$. The matching condition for $\delta \bar{E}^{(2)}$ gives us the relation for the normal interface velocity of drops in a Hele-Shaw cell,

$$
\left.U^{(0)}\right|_{n}=-\left(\delta E^{(1)}\right)_{r} .
$$

Defining $\tilde{p}=\delta E^{(1)}$, equations (5.11) (5.13) and (5.15) constitute the sharp-interface Hele-Shaw flow with electrowetting,

$$
\left.\begin{array}{l}
\Delta \tilde{p}=0, \\
\left.\tilde{p}\right|_{\partial \Omega}=A \kappa^{(0)}+B \kappa_{1}, \\
U_{n}^{(0)}=-(\tilde{p})_{r} .
\end{array}\right\}
$$

\section{Numerical simulations and discussion}

Numerical methods for solving the nonlinear Cahn-Hilliard equation are an active area of research. Barrett, Blowey \& Garcke (1999) proposed a finite element scheme to solve the fourth-order equation with degenerate mobility. In addition, the development of numerical methods for solving thin-film equations (see Zhornitskaya \& Bertozzi 2000; Grun \& Rumpf 2000; Witelski \& Bowen 2003) are also applicable to (4.7)-(4.8). We discretize the equations by finite difference in space with a semi-implicit time step,

$$
\begin{aligned}
\epsilon \frac{\rho_{n+1}-\rho_{n}}{\Delta t}+\frac{A \epsilon^{2} M}{\gamma} \Delta^{2} \rho_{n+1}=\frac{A}{\gamma}\left[\epsilon^{2} \nabla \cdot\left(\left(M-\rho_{n}\right) \nabla \Delta \rho_{n}\right)+\right. & \left.\nabla \cdot\left(\rho_{n} \nabla g^{\prime}\left(\rho_{n}\right)\right)\right] \\
& -\nabla \cdot\left(\rho_{n} \epsilon \omega\right) .
\end{aligned}
$$

We use a simple polynomial $g(\rho)=\left(\rho-\rho_{v}\right)^{2}\left(\rho-\rho_{l}\right)^{2}$. The choice of $g(\rho)$ imposes an artificial value of the liquid-vapour surface energy, $\gamma$. Integrating (4.4) gives the normalizing parameter, $\gamma=0.2322$, for the terms associated with the liquidvapour interface. All numerical results here are computed on a 256 by 128 mesh with $\Delta x=1 / 30$. The origin is located at the midpoint of the electrode edge that intersects the drop interface as shown in figure 9. The parameter $\epsilon=0.0427$ controls the diffuse-interface thickness, which is $\sim 7 \Delta x$ for all the results presented here.

A convexity splitting scheme is used where the scalar $M$ is chosen large enough to improve the numerical stability. We found $M=\max (\rho)$ serves this purpose. The equation can be solved efficiently through fast Fourier transform methods. Similar ideas were also used to simulate coarsening in the Cahn-Hilliard equation 


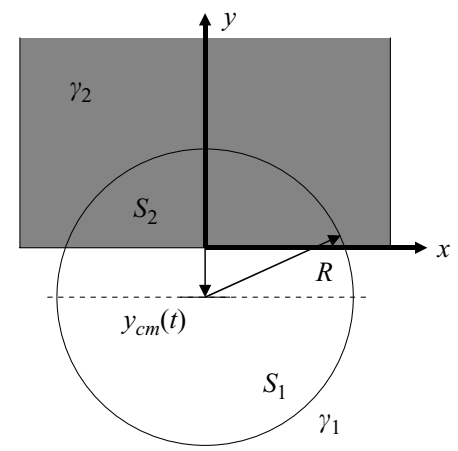

FIGURE 9. Coordinates of the numerical simulation.

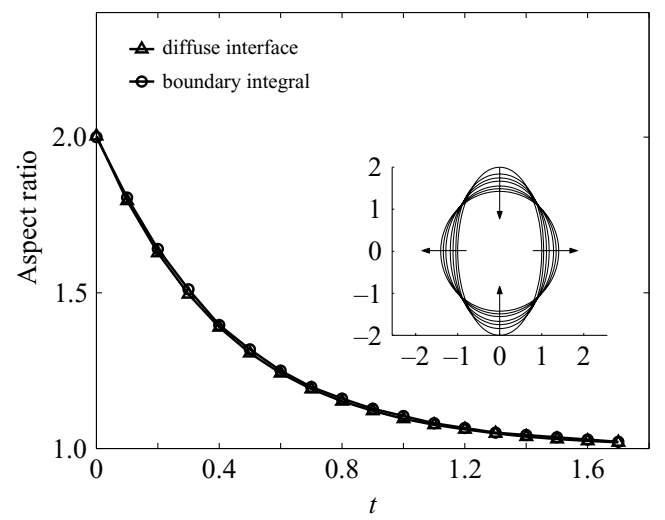

FIGURE 10. Aspect ratio of the relaxing elliptic drop calculated by the diffuse-interface model $(\triangle)$ with $\epsilon=0.0427$ and boundary integral method $(O)$.

(Vollmayr-Lee \& Rutenberg 2003) and surface diffusion (Smereka 2003). The diffuseinterface model imposes a constraint on the spatial resolution in order to resolve the transition layer, $\Delta x \leqslant C \epsilon$. Preconditioning techniques maybe implemented to relax this constraint (see Glasner 2001). We did not employ preconditioning in this study. To ensure the diffuse-interface thickness of the simulation is in the range required by the asymptotic analysis, we performed simulations with smaller values of $\epsilon$ on refined meshes and verified that the time scale of motion is independent of $\epsilon \leqslant 0.0427$.

We compare the diffuse-interface scheme to the boundary integral method by simulating the relaxation of an elliptical drop in a Hele-Shaw cell without electrowetting. Figure 10 shows a close agreement between the aspect ratios of the relaxing elliptic drops calculated by both methods.

To investigate the dynamics of an electrowetting drop without contact line dissipation, we directly compare the diffuse-interface model with $A=1$ to the electrowetting experiments using a glycerine-water drop. The experimental time and dimensions are scaled according to equation (3.7). The dimension of the square electrode is $10 \mathrm{~cm}$. The radii of the drops in figures 13 and 14 are approximately $0.55 \mathrm{~mm}$ and $0.6 \mathrm{~mm}$ respectively. Using the capacitance per area, and the surface tension of the drop reported in $\S 2$, the experimental electrowetting numbers of $\omega_{E}=7.3$ and 8.0 are derived for the drops under $50.4 \mathrm{~V} \mathrm{DC}$ voltage. 


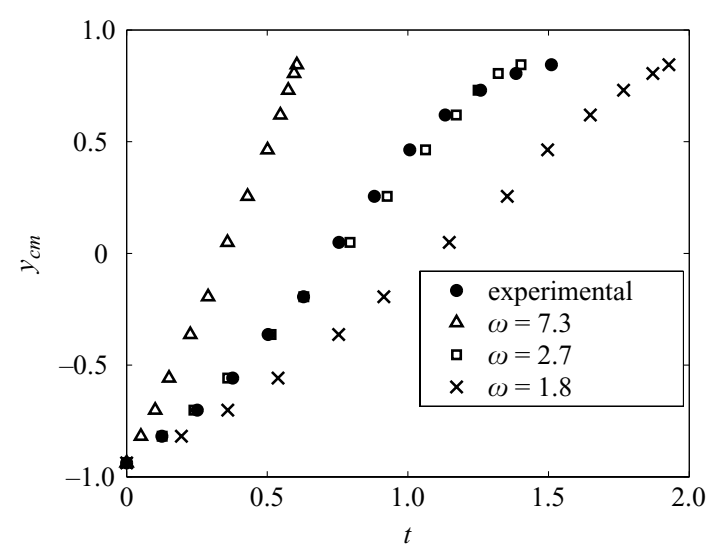

Figure 11. Positions of the centre of mass of a moving drop in experiments and in simulations at various electrowetting numbers.

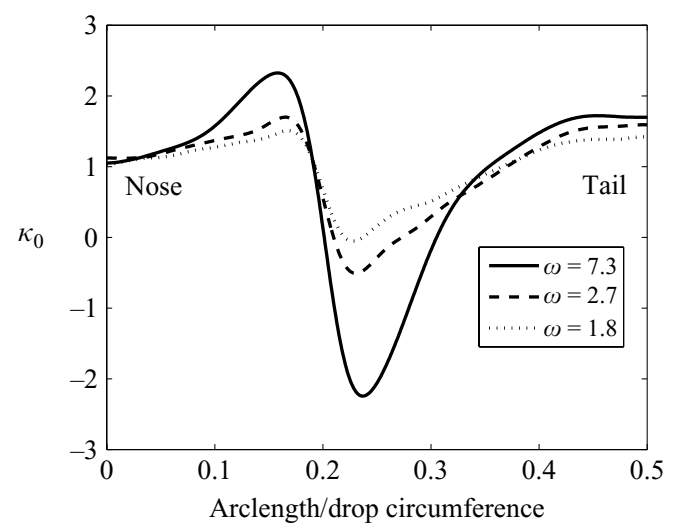

FigurE 12. Curvature along the drop contours with $y_{c m}=-0.36$ from nose to tail.

Figure 11 compares the positions of an experimental drop's centre of mass, $y_{c m}(t)$, to the simulation results at various $\omega$. Figure 13 below compares the corresponding drop contours. During the motion, electrowetting creates a necking region of negative curvature as shown in figure 12. As $\omega$ decreases, the variation of the curvature along the drop contour decreases due to the increasing influence of the surface tension. When $\omega=\omega_{E}$, the motion of the diffuse-interface drop is faster than the experiment by a factor of 2 . Scaling the electrowetting number to $\omega=\frac{3}{8} \omega_{E}$ matches the simulation with the experimental motion and produces drop contours that qualitatively agree with the experiment as shown in figure 13(c).

Figure 14(a) shows the splitting of a drop. The asymmetry of the initial drop placement yields a difference in size of the daughter drops. Figure $14(b-d)$ illustrates the capability of the method to naturally simulate the macroscopic dynamics of drop splitting. The resolution of the model is limited by the diffuse-interface thickness. Therefore we do not expect the simulation to reproduce the pinch-off of the neck and the formation of satellite drops, as seen in the last few frames of figure $14(a)$. Electrowetting initially stretches the drop, increasing the capillary pressure in the two ends and decreasing the pressure in the neck. For small $\omega$, the electrowetting cannot overcome the surface tension, which ultimately pumps the entire drop toward the end 
(a)

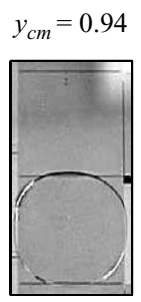

(b)

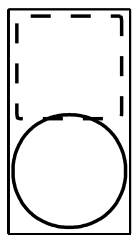

(c)

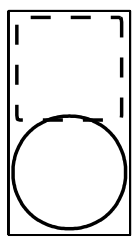

(d)

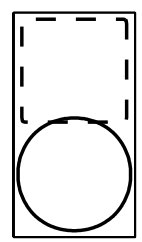

$-0.70$
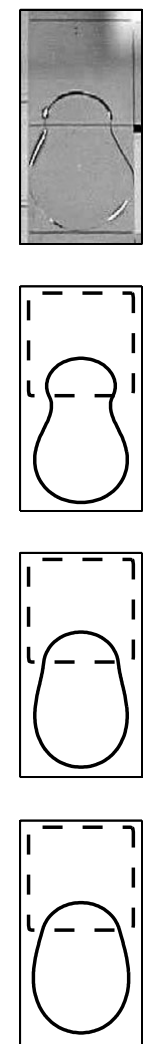

$-0.36$
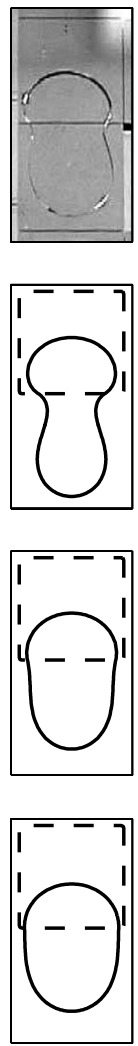

0.05
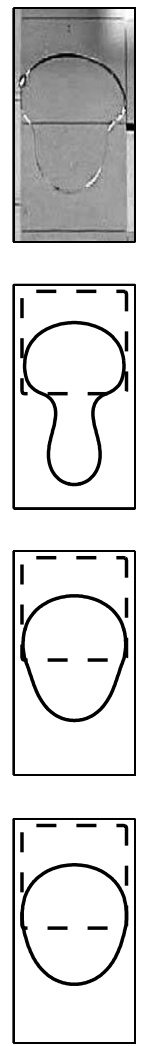

0.46
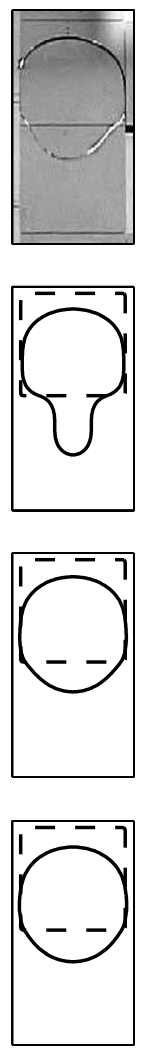

0.73
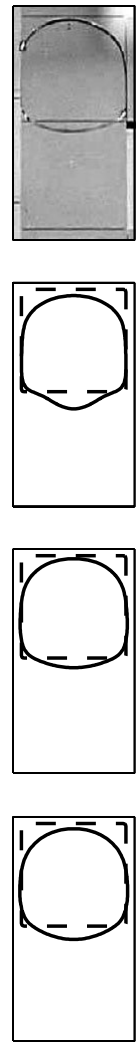

0.84
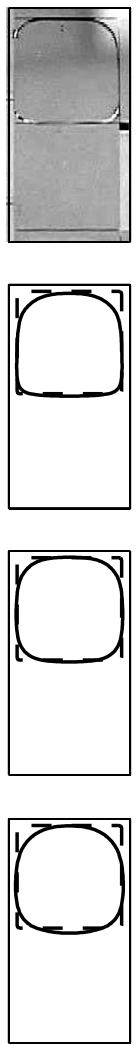

FIGURE 13. Drop movement by electrowetting: $(a)$ experimental images of the drop under $50.4 \mathrm{~V}$ of potential, $(b)$ diffuse-interface model with $\omega=7.3,(c) \omega=2.7$, and $(d) \omega=1.8$. Each column shows the drop contours with the same centre of mass position.

with smaller curvature as shown in figure $14(d)$. When $\omega=\omega_{E}$, the time scale of drop splitting is faster in the simulation than in the experiment, by a factor of 8 . In the next section, we show that the contact line dynamics has a significant influence on the electrowetting number that is more than adequate to account for this discrepancy.

\section{Contact line effect}

The previous sections investigate the drop dynamics in the absence of additional contact line effects due to the microscopic physics of the surface (see de Gennes 1985). The dynamics near the contact line results in a stress singularity at the contact line (see Huh \& Scriven 1971; Dussan V. 1979). Many have studied the contact line dynamics through various mechanisms to regularize the continuum mechanics (see Berg 1993). Inclusion of van der Waal potential in the diffuse-interface model has been proposed as a regularization of a slowly moving contact line of a partially wetting fluid (see Pomeau 2002; Pismen \& Pomeau 2004).

Implementing a full contact line model for the Hele-Shaw drop requires knowledge of several microscopic parameters. For viscous fingering in a Hele-Shaw cell, Weinstein et al. (1990) incorporated a dynamic contact angle model into the capillary pressure. However, their asymptotic analysis relies on a steady-state assumption to avoid 
(a)
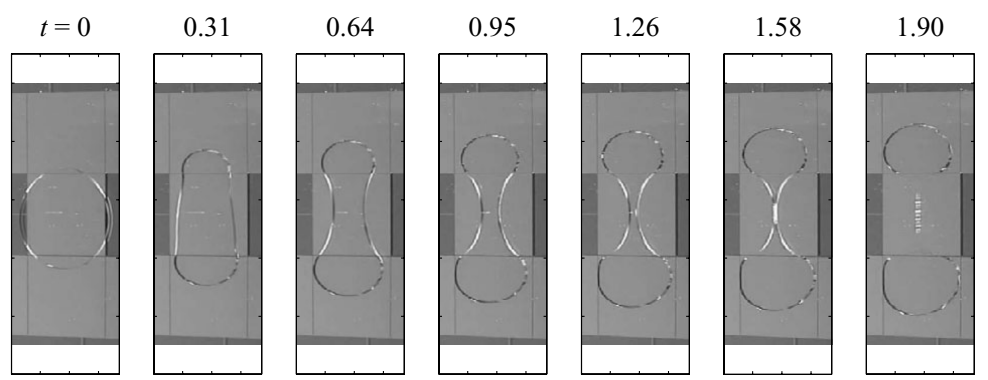

(b)
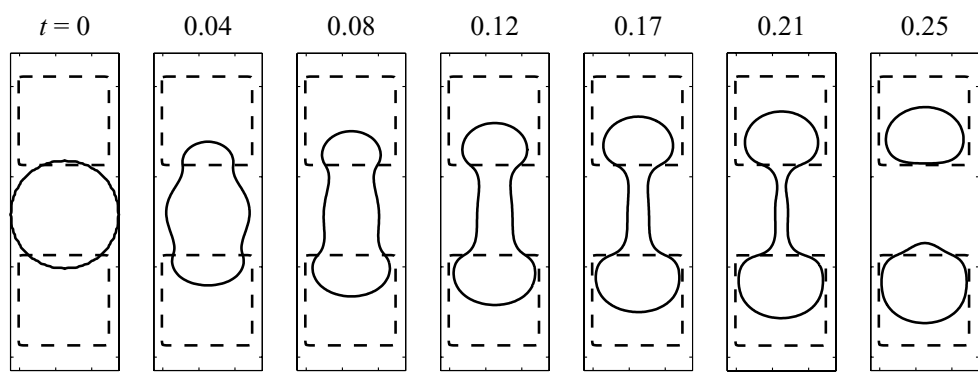

(c)
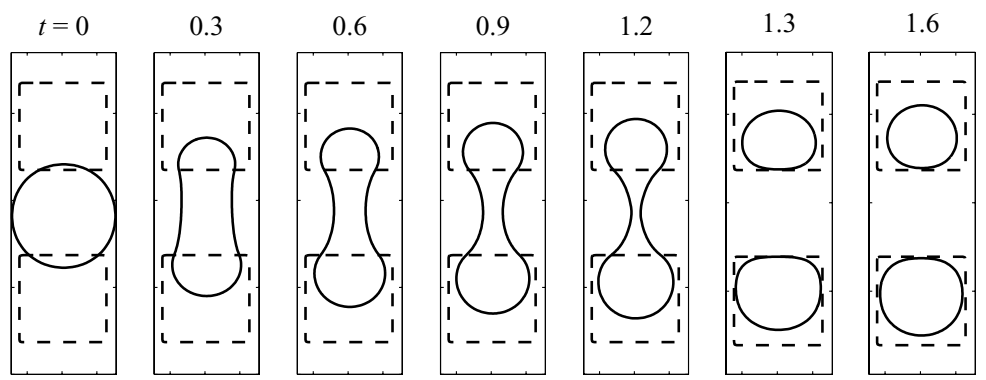

(d)
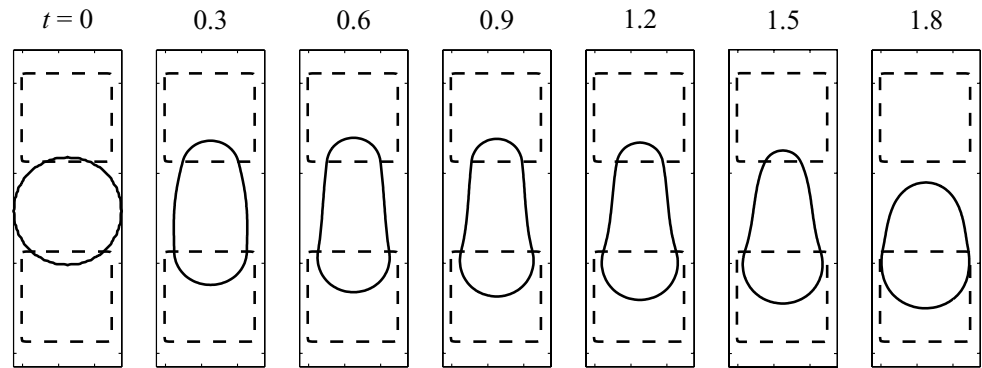

FIGURE 14. Drop splitting by electrowetting: $(a)$ images of a drop pulled apart by two electrodes under $50.4 \mathrm{~V}$ of potential, $(b)$ diffuse-interface model with $\omega=8.0,(c) \omega=3.0$, and $(d) \omega=2.0$.

solving the leading-order velocity in the inner region so the dynamic contact angle model only has geometric dependence on the finger shape. Thus their model is not directly applicable to our problem in which the interface dynamically changes in time. Therefore, how to consistently incorporate the dynamic contact angle into a general Hele-Shaw flow problem is still an interesting and open problem. Instead we estimate the influence of the contact line by a reduced-order model with fixed contact angles. Similar contact line models have been used by Ford \& Nadim (1994) and Chen et al. 


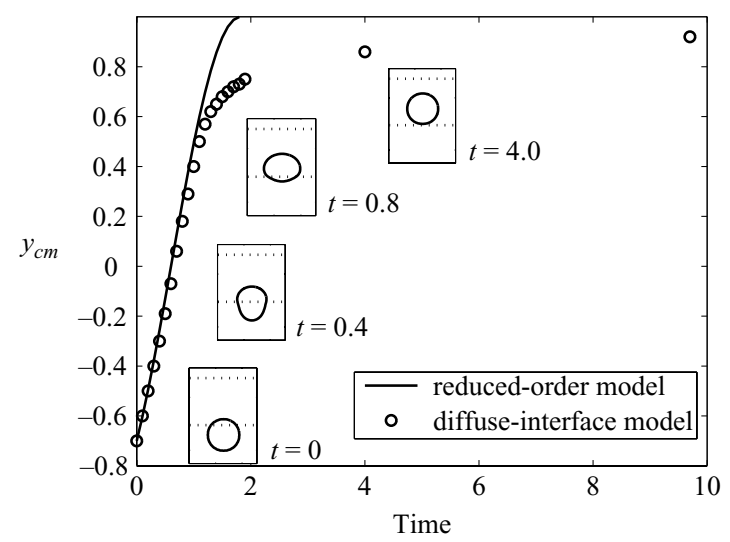

FigurE 15. The centres of mass of the reduced-order model and the diffuse-interface model. $\epsilon=0.0427, \omega=5.0$.

(2005) in the context of thermally driven drops. Incorporating a fixed contact angle into the Hele-Shaw model requires a simple extension of the incomplete displacement problem and was first discussed by Saffman (1986). In Appendix B, we carry out the necessary asymptotic expansion in the limit of small aspect ratio and extend the formulation to an asymmetric electrowetting meniscus.

The reduced-order approximation considers a drop, in the sharp-interface limit, travelling as a solid circle. This allows us to isolate the time scale of the motion from the time scale associated with the shape morphology. In Appendix A, we derive the motion of the circle's centre of mass, $y_{c m}(t)$, by considering the rate of free energy decrease,

$$
y_{c m}(t)=\sin \left(\frac{-2 \omega}{\pi} t+C\right), C=\arcsin \frac{y_{c m}(0)}{R} .
$$

Figure 15 compares the centre of mass of the reduced-order model to that of diffuseinterface model. The ability of the diffuse-interface drop to freely deform allows it to translate slightly faster into the electrowetting region. Once the entire drop mass has moved into the wetting region, a slow relaxation toward a circular shape takes place. The close agreement in the translation period shows that the diffuse-interface model does accurately simulate the gradient flow of the energy functional. The discrepancies of time scale shown in figures 13 and 14 must be attributed to additional effects in the physical problem.

The deviation of contact angles from their equilibrium values changes the capillary pressure at the interface. The contact angles increase along the advancing contact lines and decrease along the receding contact lines. The capillary pressure difference across the electrowetting and the non-electrowetting regions at the leading order becomes

$$
B \kappa_{1}=\frac{\left(\cos \theta_{r}-\cos \theta_{t}+\cos \theta_{r}-\cos \theta_{b}\right)}{\alpha},
$$

where $\theta_{r}$ is the receding contact angle on the receding contact lines. $\theta_{t}$ and $\theta_{b}$ are the advancing contact angles on the top and the bottom substrates.

In the absence of such deviations, the contact angles assume their equilibrium values, and the difference of capillary pressure is represented by the electrowetting 
number. Using (3.14) and (2.1) gives

$$
\omega=-\frac{c V^{2}}{2 \alpha \gamma_{l v}}=\frac{1}{\alpha}\left(\cos \theta_{0}-\cos \theta_{V}\right) .
$$

Comparing equations (7.2) and (7.3) gives a scaling factor

$$
\xi=\frac{B \kappa_{1}}{\omega}=\frac{\left(\cos \theta_{s}-\cos \theta_{t}\right)+\left(\cos \theta_{s}-\cos \theta_{b}\right)}{\cos \theta_{0}-\cos \theta_{V}},
$$

where $\cos \theta_{b}<\cos \theta_{V}, \cos \theta_{t}<\cos \theta_{0}$, and $\cos \theta_{r}>\cos \theta_{0}$. It can be shown that the scalar $\xi$ is less than 1 . The change of the capillary pressure may be incorporated into the reduced-order model by scaling the electrowetting number accordingly,

$$
y_{c m}(t)=\sin \left(\frac{-2 \xi \omega}{\pi} t+C\right) .
$$

Substituting the measured values of contact angles from figure 6 gives an estimate of $\xi=0.23$. This indicates that the deviation of contact angle may reduce up to $3 / 4$ of the capillary pressure difference and account for a four fold increase in the dynamic time scale.

The analysis in $\S 3$ shows that the velocity of the electrowetting drop is proportional to the difference of $B \kappa_{1}$ across the boundary of the electrowetting region. However, our estimate by the reduced-order model imposes the measured contact angles at the nose and the tail over the entire electrowetting and non-electrowetting interfaces. Therefore we expect the reduction to $1 / 4$ of the experimental electrowetting number to yield an upper bound of the time scale increase due to the contact line effect. Figure $13(c, d)$ shows that reducing $\omega$ accordingly in a simulation indeed gives a range of time scale that includes the experimental time scale. The fitting factor for $\omega$ to match the simulated motion with the experiment is $\xi=3 / 8$, well within the bound of our estimate. The failure to split the drop as shown in figure $14(d)$ further confirms that the reduction overestimates the contact line effect. The simulation results in figures $13(c)$ and $14(c)$ indicate that further refinements with a dynamic contact angle model may substantially improve the agreement with the experiment.

\section{Conclusions}

We present a diffuse-interface description of the drops in a Hele-Shaw cell in the form of a degenerate Cahn-Hilliard equation with a spatially varying surface energy. Through matching asymptotic expansions, we show that the phase-field approach approximates the sharp-interface Hele-Shaw flow in the limit of small diffuse-interface thickness. The dynamics in the sharp-interface limit is validated numerically by a direct comparison to the boundary integral methods. This approach enables us to naturally simulate the macroscopic dynamics of drop splitting, merging, and translation under the influence of local electrowetting.

As illustrated by the reduced-order model, the contact line dynamics significantly affects the problem by modifying the cross-substrate of the interface. Based on the measured advancing and receding contact angles, we showed that the strong influence of contact line dynamics accounts for up to a four fold increase in the dynamic time scale of the Hele-Shaw approximation. This points out that using the Lippman equation under the assumption of quasi-steady interfacial motion is not adequate to capture the physics at the interface of the electrowetting drop. Knowledge of the interface geometries near the electrowetting boundary may improve our estimate. 
Numerical simulations of drop motions showed a range of dynamic time scale that is consistent with the experimentally measured time scale.

The assumption of constant advancing and receding contact angles in the reducedorder model is obviously a crude approximation. Improvements to this approximation need to account for the dynamically varying contact angle since the normal velocity of the entire drop interface varies. Careful characterizations of the electrowetting contact line dynamics and asymptotic matching to the bulk Hele-Shaw flow must be considered to determine an appropriate pressure boundary condition. In short, this will introduce velocity dependence in the formulation of $B\left(\omega,\left.C a\right|_{\partial \Omega}\right)$ in equation (3.10), where $\left.C a\right|_{\partial \Omega}$ is the local capillary number of the interface. To formulate $B\left(\omega,\left.C a\right|_{\partial \Omega}\right)$, the asymptotic analysis of Weinstein et al. (1990) must be extended from the special case of a travelling wave solution to the general motion of a Hele-Shaw drop. Doing so presents an interesting problem for future research.

The coupling between pressure boundary condition and the local contact line velocity can be accomplished by modifying (4.7)-(4.8) to a viscous degenerate CahnHilliard equation (see Seppecher 1996) of the form

$$
\begin{aligned}
\epsilon \rho_{t}+\epsilon^{2} \nabla \cdot\left(\rho \nabla f\left(\rho_{t}\right)\right) & =\nabla \cdot(\rho \nabla(\delta E)), \\
\delta E & =\frac{A}{\gamma}\left(-\epsilon^{2} \Delta \rho+g^{\prime}(\rho)\right)-\epsilon \omega,
\end{aligned}
$$

where $f\left(\rho_{t}\right)$ is the diffused-interface analogue of the sharp-interface dynamic contact angle model, $B\left(\omega,\left.C a\right|_{\partial \Omega}\right)$. The additional nonlinearity in equation (8.1) changes the properties of the numerical approximation dramatically and warrants new numerical research efforts to efficiently solve it. A matched asymptotic expansion similar to the one in $\S 5$ shows that the addition of $f\left(\rho_{t}\right)$ introduces a contact line velocity dependence into the pressure boundary condition.

The threshold voltage for moving a drop shown in figure 4 indicates hysteresis may also be a significant source of dissipation. The MEMS fabrication process for the electrowetting devices produces variations of the surface energy that must be overcome by the moving contact line. The dissipation becomes more significant as the drop radius decreases, such as in microfluidic applications. Even with the length scale of our experiment $(\sim 1 \mathrm{~cm})$, the surface effect may become important for specific motions such as the splitting of a partially wetting drop. A preliminary study shows the neck width at pinch-off is much smaller than the separation height, $b$. This behaviour is different from experiments mentioned in Constantin et al. (1993), suggesting that the surface effect may delay the capillary pinch-off by preventing the fluid neck from separating off the substrates. For a drop in motion, the linear relationship of the drop velocity in figure 4 suggests that the dissipation by the contact angle hysteresis can be accounted for by an offset of the square of the voltage. However, the range of linear behaviour is not sufficient to validate this hypothesis. To further study the effect of hysteresis on the time scale of motion, additional microscopic experiments are needed to characterize the strength and the uniformity of the defects on the substrates. This information will allow us to introduce a macroscopic model of the microscopic physics, such as the work of Joanny \& Robbins (1990), into the study of electrowetting drops.

Another interesting problem is that of a viscous drop of larger aspect ratio. The velocity component normal to the substrates becomes significant. In this case, the two-dimensional Hele-Shaw model can no longer provide an adequate approximation. On the other hand, a fully three-dimensional simulation of such a drop is an extremely 
complicated task. It would be desirable to develop a reduced-dimension model that is computationally tractable while preserving the essential information about the velocity component normal to the substrates. Galerkin methods from numerical analysis may provide an interesting direction to accomplish this.

We thank Pirouz Kavehpour for invaluable experimental support, and discussions on contact line dynamics. We gratefully acknowledge valuable discussions of HeleShaw cells with George M. Homsy, and Sam D. Howison. We also thank Hamarz Aryafar and Kevin Lu for their expertise and assistance in the use of high speed camera and rheometer. This is work is supported by ONR grant N000140710431, NSF grant CI-0321917, NSF grant DMS-0405596, and NASA through Institute for Cell Mimetic for Space Exploration (CMISE).

\section{Appendix A. Reduced-order model}

Consider a semi-infinite electrowetting region; we approximate the drop motion as a moving solid circle. Using the lubrication approximation, we balance the rate of viscous dissipation with the rate of free energy decrease,

$$
D \approx-\frac{b^{3}}{12 \mu} \int_{R^{2}} \rho|\nabla p|^{2} \mathrm{~d} x \mathrm{~d} y=-\frac{12 \mu}{b} \int_{R^{2}} \rho|\boldsymbol{U}|^{2} \mathrm{~d} x \mathrm{~d} y=\frac{\mathrm{d} E}{\mathrm{~d} t} .
$$

The centre of the circle travels along the axis as shown in figure 9. The distance between the boundary of the electrowetting region and the origin is $d$. The position of the centre is $y_{0}(t)$ where $y_{0}(0)=0$. The drop moves as a solid circle so the integral reduces to

$$
-\frac{12 \mu\left|\dot{y}_{c m}\right|^{2} \pi R^{2}}{b}=\frac{\mathrm{d} E}{\mathrm{~d} t},
$$

where $\dot{y}_{c m}$ denotes the velocity of the centre of the circle. The free energy is composed of the surface energy of the dielectric surface with no voltage applied $\gamma_{1}$, the surface energy of the electrowetting region $\gamma_{2}$, and the liquid-vapor surface energy. Since the liquid-vapor interface area remains constant, the rate of change in free energy is

$$
\frac{\mathrm{d} E}{\mathrm{~d} t}=\Delta \gamma \dot{S}_{2},
$$

where $\Delta \gamma=\gamma_{2}-\gamma_{1}$ and $\dot{S}_{2}$ is the derivative of the drop area inside the electrified region with respect to time

$$
\dot{S}_{2}=-2 R^{2} \sqrt{1-y_{c m}^{2}} \dot{y}_{c m}
$$

Equations (A 2), (A 3), and (A 4)give the following ODE:

$$
\dot{y}_{c m}=\frac{b \Delta \gamma}{6 \mu \pi R^{2}} \sqrt{1-y_{c m}^{2}} .
$$

Integrating this ODE we obtain

$$
y_{c m}(t)=\sin \left(\frac{b \Delta \gamma}{6 \mu \pi R^{2}} t+C\right), C=\arcsin \frac{y_{c m}(0)}{R},
$$

If we neglect the surface effects, the energy difference between the two regions is well-described by (1.1), $\Delta \gamma=-c V^{2} / 2$. After changing time to a dimensionless 
(a)

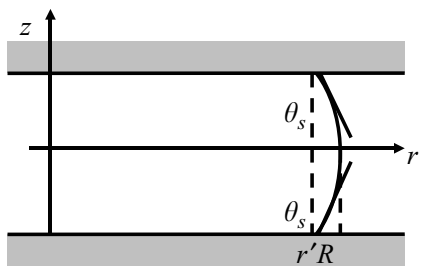

(b)

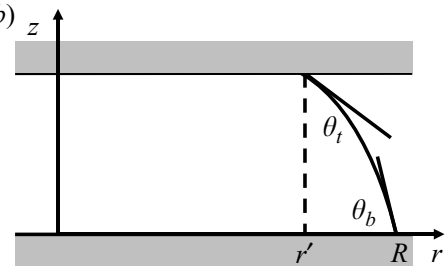

FIGURE 16. Coordinates and geometry of $(a)$ a symmetric interface and $(b)$ an asymmetric interface due to electrowetting.
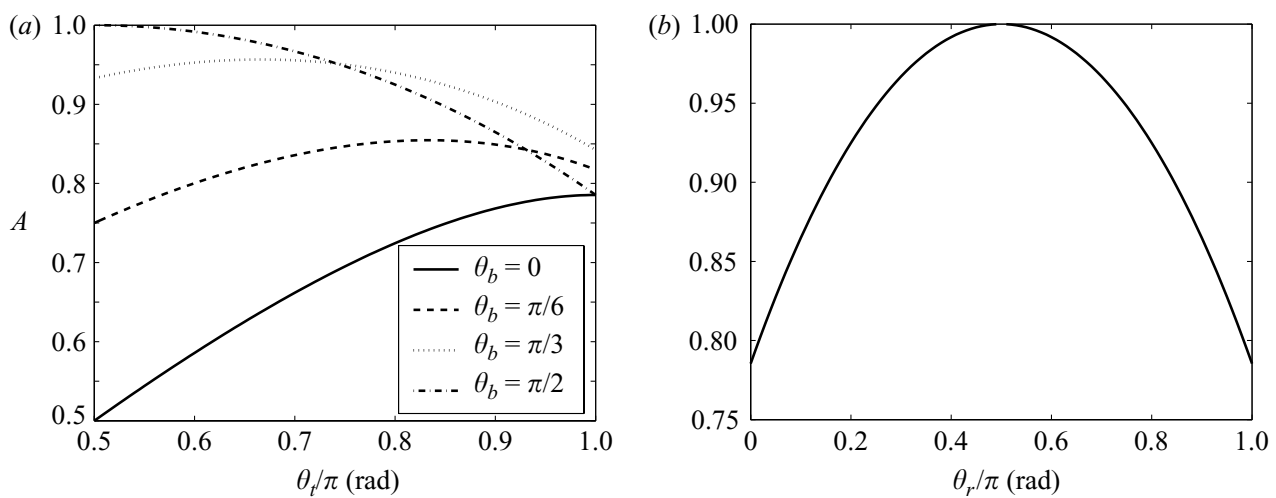

FIGURE 17. Horizontal curvature $A$ for $(a)$ electrowetting and $(b)$ non-electrowetting menisci.

variable, we obtain

$$
y_{c m}(t)=\sin \left(\frac{-2 \omega}{\pi} t+C\right) \text {. }
$$

\section{Appendix B. Interfacial condition}

Here we relate the pressure boundary condition (3.3) to the local contact angle by perturbation expansions of the governing equations with respect to the aspect ratio by considering a radially expanding partially wetting fluid (see Saffman 1986). We calculate the conditions for both the symmetric interfaces and the asymmetric interfaces. The leading-order expansion then relates the cross-substrate curvature, $B \kappa_{1}$, to the local contact angle, and the next-order expansion corresponds to the contribution from the horizontal curvature, $A \kappa_{0}$.

Outside the electrowetting region, the interface is symmetric as shown in figure $16(a) ; z=h(r)$ gives the shape of the interface. The pressure boundary conditions are the only relevant equations to solve up to $O(\alpha)$. The equation at the leading order is integrated twice with boundary conditions $h_{r}^{0}(R)=-\infty$ and $h^{0}(R)=0$ to obtain $h^{0}(r)$ Then apply the boundary conditions that $h^{0}\left(r^{\prime}\right)=1$, where $r^{\prime}=\left(1-\sin \theta_{s}\right) / \cos \theta_{s}+R$ to obtain the leading-order pressure. The next order is solved by a similar procedure with the boundary conditions $h_{r}^{1}(R)=0, h^{1}(R)=0$ and $h^{1}\left(r^{\prime}\right)=0$ :

$$
\left.P\right|_{\partial \Omega}=\frac{-2 \cos \theta_{s}}{\alpha}-\frac{1+\sin \theta_{s}}{\cos \theta_{s}}\left(\frac{\theta_{s}}{2}-\frac{\pi}{4}\right)+O(\alpha) .
$$

Inspection of the $O(\alpha)$ term, plotted in figure $17(b)$, shows that $A$ varies between a maximum of 1 when $\theta_{s}=\pi / 2$ and a minimum of $\pi / 4$ when $\theta_{s}=0$ and $\pi$. 
If the interface inside the electrowetting region satisfies the requirements that $\theta_{t} \geqslant \pi / 2$ and $\theta_{b} \leqslant \pi / 2$ as shown in figure $16(b)$, we can perform similar expansions for an interface inside the electrowetting region with a shifted coordinate. At the leading order, we apply the boundary conditions $h_{r}^{0}(R)=-\tan \theta_{b}, h^{0}(R)=0$ and $h^{0}\left(r^{\prime}\right)=1$, where $r^{\prime}=-\left(\sin \theta_{t}-\sin \theta_{s}\right) /\left(\cos \theta_{t}+\cos \theta_{b}\right)+R$. The next order is solved with the boundary conditions $h_{r}^{1}(R)=0, h^{1}(R)=0$ and $h^{1}\left(r^{\prime}\right)=0$ :

$$
\left.P\right|_{\partial \Omega}=\frac{-\left(\cos \theta_{b}+\cos \theta_{t}\right)}{\alpha}-\frac{\cos \theta_{t}}{2\left(1-\cos \left(\theta_{b}-\theta_{t}\right)\right)}\left(\frac{\cos \theta_{b}}{\cos \theta_{t}} \sin \left(\theta_{b}-\theta_{t}\right)+\theta_{t}-\theta_{b}\right)+O(\alpha) .
$$

Taking the leading-order difference between the two interfaces gives the same formula as equation (7.2). For the interface inside the electrowetting region, figure 17(a) shows $A$ varying between a maximum value of 1.0 when $\theta_{b}=\theta_{t}=\pi / 2$ and a minimum of 0.5 at $\theta_{b}=0$ and $\theta_{t}=\pi / 2$. Since relaxation dominates away from the boundary of the electrowetting region, we can obtain good estimate of $A$ by substituting the data in figure 6. The computed values of $A$ are 0.9994 and 0.9831 for the interfaces outside and inside the electrowetting region respectively. This supports the use of $A=1$.

\section{REFERENCES}

Barrett, J. W., Blowey, J. F. \& Garcke, H. 1999 Finite element approximation of the Cahn-Hilliard equation with degerate mobility. SIAM J. Numer. Anal. 37, 286-318.

Bensimon, D., Kadanoff, L. P., Liang, S. \& Shraiman, S. 1986 Viscous flow in two dimensions. Rev. Mod. Phys. 58, 977-999.

BERG, J. C. (Ed.) 1993 Wettability. Marcel Dekker.

Bretherton, F. P. 1961 The motion of long bubble in tubes. J. Fluid Mech. 10, 166-188.

CaginalP, G. \& Fife, P. 1988 Dynamics of layered interfaces arising from phase boundaries. SIAM J. Appl. Maths 48, 506-518.

Carrillo, L., Soriano, J. \& Ortin, J. 1999 Radial displacement of a fluid annulus in a rotating Hele-Shaw cell. Phys. Fluids 11, 778-785.

Carrillo, L., Soriano, J. \& Ortin, J. 2000 Interfacial instabilities of a fluid annulus in a rotating Hele-Shaw cell. Phys. Fluids 12, 1685-1698.

Chen, J. Z., Troian, S. M., Darhuber, A. A. \& Wagner, S. 2005 Effect of contact angle hysteresis on thermocapillary droplet actuation. J. Appl. Phys. 97, 014906.

Cho, S. K., Moon, H., Fowler, J., Fan, S.-K. \& Kim, C.-J. 2001 Splitting a liquid droplet for electrowetting-based microfluidics. In Proc. ASME IMECE, Paper. 2001-23831.

Cho, S.-K., Moon, H. \& KIM, C.-J. 2003 Creating, transporting, cutting, and merging liquid droplets by electrowetting-based actuation for digital microfluidic circuits. J. Microelectromech. Syst. 12, 70-80.

Constantin, P., Dupont, T. F., Goldstein, R. E., Kadanoff, L. P., Shelley, M. J. \& Zhou, S.-M. 1993 Droplet breakup in a model of the Hele-Shaw cell. Phys. Rev. E 47, 4169-4181.

Darhuber, A. \& Troian, S. M. 2005 Principles of microfluidic actuation by manipulation of surface stresses. Annu. Rev. Fluid Mech. 37, 425-455.

De Gennes, P. G. 1985 Wetting: statics and dynamics. Rev. Mod. Phys. 57, 827-863.

Dussan, V. E. B. 1979 On the spreading of liquid on solid surfaces: static and dynamic contact lines. Annu. Rev. Fluid Mech. 11, 371-400.

Ford, M. L. \& NADIM, A. 1994 Thermocapillary migration of an attached drop on a solid surface. Phys. Fluids 6, 3183-3185.

Glasner, K. 2001 Nonlinear preconditioning for diffuse interfaces. J. Comput. Phys. 174, 695-711.

Glasner, K. 2003 A diffuse interface approach to Hele-Shaw flow. Nonlinearity 16, 49-66.

Grun, G. \& RUMPF, M. 2000 Nonnegativity preserving convergent schemes for the thin film equation. Numer. Maths 87, 113-152. 
Hayes, R. A. \& Feenstra, B. J. 2003 Video-speed electronic paper based on electrowetting. Nature 425, 383-385.

Hele-Shaw, H. J. S. 1898 The flow of water. Nature 58, 34-36.

Homsy, G. M. 1987 Viscous fingering in porous media. Annu Rev. Fluid Mech. 19, 271-311.

Hou, T., Lowengrub, J. S. \& Shelley, M. J. 1994 Removing the stiffness from interfacial flow with surface-tension. J. Comput. Phys. 114, 312-338.

Howison, S. D. 1992 Complex variable methods in Hele-Shaw moving boundary problems. Eur. J. Appl. Maths 3, 209-224.

Huh, C. \& SCRIVEN, L. E. 1971 Hydrodynamic model of steady movement of a solid-liquid-fluid contact line. J. Colloid Interface Sci. 35, 85-101.

JoAnny, J.-F. \& Robbins, M. 1990 Motion of a contact line on a heterogenous surface. J. Chem. Phys. 92, 3206-3212.

KANG, K. H. 2002 How electrostatic fields change contact angle in electrowetting. Langmuir 18, $10318-10322$.

KIM, C.-J. 2000 Microfluidics using the surface tension force in microscale. In SPIE Symp. Micromachining and Microfabrication, vol. 4177, pp. 49-55. Santa Clara, CA.

Koнn, R. V. \& Отто, F. 1997 Small surface energy, coarse-graining, and selection of microstructure. Physica D 107, 272-289.

Lee, H., Lowengrub, J. S. \& Goodman, J. $2002 a$ Modeling pinchoff and reconnection in a HeleShaw cell. I. The models and their calibration. Phys. Fluids 14, 492-513.

LEE, J. \& KIM, C.-J. 1998 Liquid micromotor driven by continuous electrowetting. In IEEE Micro ElectroMechanical Systems Workshop, pp. 538-543, Heidelberg, Germany.

Lee, J., Moon, H., Fowler, J., Schoellhammer, J. \& Kim, C.-J. 2001 Addressable micro liquid handling by electric control of surface tension. In Proc. IEEE Conf. Micro ElectroMechanical Systems, pp. 499-502, Interlaken, Switzerland.

Lee, J., Moon, H., Fowler, J., Schoellhammer, J. \& Kim, C.-J. $2002 b$ Electrowetting and electrowetting-on-dielectric for microscale liquid handling. Sensors Actuators A 95, 259-268.

Lippman, M. G. 1875 Relations entre les phènoménes électriques et capillaires. Ann. Chim. Phys. 5, 494-548.

Moon, H., Cho, S.-K., Garrel, R. L. \& Kim, C.-J. 2002 Low voltage electrowetting-on-dielectric. J. Appl. Phys. 92, 4080-4087.

Mugele, M. \& Baret, J.-C. 2005 Electrowetting: from basics to applications. J. Phys.: Condens. Matter 17, R705-R774.

Отто, F. 1998 Dynamics of labyrinthine pattern formation in magnetic fluids: A mean-field theory. Arch. Rat. Mech. Anal. 141, 63-103.

Park, C.-W. \& Homsy, G. M. 1984 Two-phase displacement in Hele-Shaw cells: theory. J. Fluid Mech. 139, 291-308.

Paterson, A. \& Fermigier, M. 1997 Wetting on heterogeneous surfaces: Influence of defect interactions. Phys. Fluids 9, 2210-2216.

Paterson, A., Fermigier, M. \& Limat, L. 1995 Wetting on heterogeneous surfaces: Experiments in an imperfect hele-shaw cell. Phys. Rev. E 51, 1291-1298.

Pego, R. L. 1989 Front migration in the nonlinear cahn hilliard equation. Proc. R. Soc. Lond. A 422, 261-278.

Peykov, V., Quinn, A. \& Ralston, J. 2000 Electrowetting: a model for contact-angle saturation. Colloid Polym. Sci. 278, 789-793.

Pismen, L. M. \& Pomeau, Y. 2004 Mobility and interactions of weakly nonwetting droplets. Phys. Fluids 16, 2604-2612.

Pollack, M. G., Fair, R. B. \& Shenderov, A. D. 2000 Electrowetting-based actuation of liquid droplets for microfluidic applications. Appl. Phys. Lett 77, 1725-1726.

PomeaU, Y. 2002 Recent progress in the moving contact line problem: a review. C. R. Mechanique 330, 207-222.

ReInelt, D. A. 1987 Interface conditions for two-phase displacement in Hele-Shaw cells. J. Fluid Mech. 184, 219-234.

Saffman, P. G. 1986 Viscous fingering in Hele-Shaw cells. J. Fluid Mech. 173, 73-94.

SAfFman, P. G. \& TAYLOR, G. I. 1959 A note on the motion of bubbles in a Hele-Shaw cell and porous medium. Q. J. Mech. Appl. Maths 12, 265-279.

SePpecher, P. 1996 Moving contact lines in the Cahn-Hilliard theory. Intl J. Engng Sci. 34, 977-992. 
Seyrat, E. \& Hayes, R. A. 2001 Amorphous fluoropolymers as insulators for reversible low-voltage electrowetting. J. Appl. Phys. 90, 1383-1386.

Shapiro, B., Moon, H., Garrell, R. L. \& Kim, C.-J. 2003 Equilibrium behavior of sessile drops under surface tension, applied external fields, and material variations. J. Appl. Phys. 93, 5794-5811.

SMEREKA, P. 2003 Semi-implicit level-set method for curvature and surface diffusion motion. J. Sci. Comput. 19, 439-456.

TANVEer, S. 2000 Surprises in viscous fingering. J. Fluid Mech. 409, 273-308.

Vallet, M., Vallade, M. \& Berge, B. 1999 Limiting phenomena for the spreading of water on polymer films by electrowetting. Eur. Phys. J. B 11, 583-591.

Verheijen, H. J. J. \& Prins, M. W. J. 1999 Reversible electrowetting and trapping of charge: model and experiments. Langmuir 15, 6616-6620.

Vollmayr-Lee, B. P. \& Rutenberg, A. D. 2003 Fast and accurate coarsening simulation with an unconditionally stable time step. Phys. Rev. E 68, 066703.

WALKer, S. \& Shapiro, B. 2006 Modeling the fluid dynamics of electro-wetting on dielectric (EWOD). J. Microelectromech. Syst. 15, 986-1000.

Weinstein, S. J., Dussan, V. E. B. \& Ungar, L. H. 1990 A theoratical study of two-phase flow through a narrow gap with a moving contact line: viscous fingerin in a Hele-Shaw cell. J. Fluid Mech. 221, 53-76.

Witelski, T. P. \& Bowen, M. 2003 Adi schemes for higher-order nonlinear diffusion equations. Appl. Numer. Math. 45, 331-351.

Yeo, L. Y. \& Chang, C. H. 2006 Electrowetting films on parallel line electrodes. Phys. Rev. E 73, 011605-011616.

Zhornitskaya, L. \& Bertozzi, A. L. 2000 Positivity-preserving numerical schemes for lubricationtype equations. SIAM J. Numer. Anal. 37, 523-555. 